\title{
Frontières Et Dynamiques Socio-Spatiales En Afrique : Une Analyse À Partir Des Frontières Sud- Camerounaises
}

\author{
Abdoulay Mfewou (PhD) \\ Hervé Tchekote (PhD) \\ Josephine Lemouogue (PhD) \\ University of Dschang-Cameroun
}

Doi: 10.19044/esj.2018.v14n5p285 URL:http://dx.doi.org/10.19044/esj.2018.v14n5p285

\begin{abstract}
This article focuses on the openness and socio-economic dynamics associated with the meeting of different communities from many inland territories of Africa: on the borders of South Cameroon, northern Equatorial Guinea and North of Gabon, attracted by the oil windfall. The study illustrates the effectiveness of "living together" in this vast sub-region. It shows how development is based on the organization of trade that is not simply based on the differences between national systems, but on the establishment of SouthSouth relations. While the challenges based on planning on these cross-border spaces is indispensable, local elected representatives are trying to set-up networking arrangements for cross-border communities. Border management makes it possible to ensure security, counter-threats to people and economic prosperity. The construction of a motorable high-way and the implementation of treaties on the opening of borders signed between the States since the 16th of March 1994 deemed at facilitating the free movement of economic actors, while many still consider the arrival of foreigners in these Eldorado as an invasion.
\end{abstract}

Keywords: Social dynamics, Boundaries, zones forester-Cameroon

Résumé

Cet article montre le degré d'ouverture et de dynamisme socioéconomique d'enseignements sur l'effectivité de vivre ensemble entre les différents communautés venus de partout à l'intérieur de l'Afrique et est attiré surtout par la manne pétrolière de la Guinée Equatoriale et celle du Gabon. L'enjeu d'aménagement pour ces espaces transfrontaliers consiste à développer des échanges commerciaux qui ne reposent pas simplement sur le différentiel entre les systèmes nationaux mais surtout dans la relation Sud-Sud, 
les élus locaux souhaitent ouvertement le jumelage des communes transfrontalières, la gestion commune des frontière à assurer la sécurité pour contrer les menaces à la sûreté et à la prospérité économique, la construction de l'autoroute et la mise en pratique des traités d'ouverture des frontières signés entre les États depuis le 16 mars 1994, qui pourra faciliter la libre circulation des acteurs légitimes bien que vivant au frein de peur de l'invasion des étrangers dans ces Eldorado.

Mot-clés: Dynamique sociale, Frontière, zone forestière-Cameroun

\section{Introduction}

Dans une dynamique économique transfrontalière (Diallo M. M., 2014; Christian Bouquet et al., 2007), le Sud du Cameroun est devenue une ressource qui attire et qui mobilise les populations d'origines diverses, venues des pays d'Afrique. Ces échanges s'inscrivent dans une mondialisation de la relation Sud-Sud et ont un impact significatif sur la consolidation du développement sous régional et de l'insertion transfrontalière souhaitée par l'Union Africaine (libre circulation pour tous).

Le Cameroun, pays d'Afrique Centrale partage ses frontières terrestres avec six pays voisins, le Nigeria, le Tchad, la République centrafricaine, la République du Congo, le Gabon et la Guinée équatoriale, pour un total de $4591 \mathrm{~km}$. Mais, avec l'exploitation du pétrole au Gabon et en Guinée Equatoriale, les mobilités entre le Cameroun et les pays limitrophes de sa frontière Sud se sont intensifiés, le Sud faisant l'objet d'une attraction particulière. En effet, l'attractivité de la zone s'est amplifiée avec la production pétrolière du Golfe de Guinée qui a fourni plus de 4,5\% de la production mondiale (IMF / GABON., 2016). De petites villes et des lieux de marché se sont structurée linéairement tout long des réseaux de transports, dominés par les villes de Kyé-Ossi, de Abang-Minko'o et de Ebebiyin, puis conduisant aux grandes villes capitales du Gabon (Libreville) et de la Guinée Equatoriale (Malabo).

Ce développement économique s'accompagne d'un flux commercial entre différents pays, mis en place par des acteurs diversifiés (Igué, J. O., 2010 et Hugon, Ph., 1989). Pourtant, il est à remarquer que cette frontière, même si elle est une ressource économique, reste une réalité éminemment politique. Elle s'impose comme une limite de souveraineté et de compétence territoriale pour décrire le processus historique de colonisation du territoire (Lévy et Lussaul - 2003). Il est important de souligner que la frontière camerounoguinée équatoriale est un exemple typique de frontière issue de la rivalité coloniale franco-espagnole. Ici, comme d'ailleurs dans bon nombre de régions frontalière d'Afrique, de très nombreuses ethnies se trouvent séparées par la ligne frontalière léguée par la colonisation. 
Par ailleurs, la zone frontalière Sud camerounaise est partagée entre des parcs naturels, notamment Mengam, Minkébé et Del Monte Temelon, et des bassins fluviaux tels que Woleu Ntem et Tala-tala. En effet, la ligne frontalière forestière terrestre entre le Cameroun et la République du Congo $(523 \mathrm{~km})$, le Gabon $(298 \mathrm{~km})$ et la Guinée équatoriale $(189 \mathrm{~km})$ s'étend dans une forêt dense jouant un rôle écologique important.

Dans cet ensemble naturel, apparemment homogène, se jouent des jeux de vivre ensemble, l'intégration et les dynamiques marchandes (Nkendah, R., 2011 et Bouquet, C., 2003). Car, cette frontalière entre les pays voisins est accompagnée étroitement d'un espace dans une dynamique sociale et économique, combinant discontinuité et cohésion territoriales aux confins étatiques du Nord de la Guinée équatoriale, du Nord du Gabon, et du Nord du Congo Brazzaville. On y trouve de nombreux groupes ethniques dont la cohabitation apparait comme une ressource, créant les marchés dits périphériques, dont ceux de Kyé-Ossie, Abang-Minko'o et Ebebiyin. Des jeux de mobilité se construisent autour d'un dispositif remarquable de contrôle de visa d'entrée mis en place pour gérer la circulation des individus.

Ces constats nous amènent à poser les questions essentielles dans ce contexte de mobilité. Quelles sont les dynamiques socio-spatiales en cours au niveau de la frontière Sud camerounaise ? Quelles sont les mutations en termes de développement qui en découlent ? Pour répondre à ces interrogations, notre méthodologie a consisté de mener une enquête de terrain auprès des différents acteurs. Nous avons interrogé des responsables des communes, des membres des populations marchandes (1390 hommes contre 2807 femmes) concernant leur profit et nous avons enregistré une typologie d'acteurs. Nous avons aussi analysé les initiatives de la gouvernance transfrontalière de chaque pays à partir des sources écrites et de son application sur le terrain. L'analyse des caractéristiques sociodémographique, mais aussi de la structuration des territoires au niveau des frontières Sud camerounaises a constitué le schéma de ce travail.

\section{Les caractéristiques démographiques et Dynamique sociale et Sécuritaire à la frontière Sud Camerounaise}

Concentrant une population cosmopolite au confluent de quatre Etats, la frontière Sud camerounaise fait partie des régions les plus faiblement peuplés (20-30 habitants/km2) du Cameroun. Le département de la Vallée-duNtem rassemble 64747 habitants sur une superficie de $7303 \mathrm{~km} 2$ avec la commune Kyé-Ossi qui comptait en 2005, 17127 habitants lors du dernier recensement, la commune d'Olamze de 8518 habitants, la commune d'Ambam de 15964 habitants et la commune de Ma'an de 904 habitants. Une solidarité étroite s'est établie entre les Hommes et la forêt et les relations sont souvent stables avec les habitants des commune des pays voisins de 
périphérique : la province Woleu-Ntem, l'une des neuf provinces du Gabon avec son chef-lieu Oyem qui rassemble les villes de Bitam, Minvoul, Mitzic et Médouneu, avec 157000 habitants (recensements de 2010).

La Guinée équatoriale collée à cette ligne frontalière est constituée de deux parties, l'une continentale, bordée par le Cameroun et le Gabon. Sa province Kie Ntem partage la ligne frontalière-camerounaise. Elle a pour cheflieu Ebebiyin, où s'est créé un marché limitrophe. Les familles des deux côtés gardent parfois les mêmes arbres généalogiques (Fang Mabéa, Fang Tumu...). Cet espace (figure 1) est faiblement peuplé et d'ethnies mêlées (Fang, BétiPahouin, Baka...). S'y rencontrent les caractères des situations sociodémographiques contrastées des différents groupes venus d'autres régions des trois Etats, qui ont des niveaux de développement variés. KyéOssi, Abang-Minko'o et Ebebiyin offrent un climat favorable aux affaires pour la population locale et les étrangers (nigérian, tchadien, malien, sénégalais...). On enregistre une intense activité commerciale à partir de la mise en place de stratégies des communes, générant la circulation des personnes et des biens, dont celles des commerçants et des consommateurs qui se déplacent de part et d'autre de la frontière pour vendre et pour acheter des produits agricoles et des produits de premiers nécessités.

Cette mutation commerciale frontalière fait suite au boom pétrolier qu'a connu le Gabon et la Guinée Equatoriale. Même avec le prix du pétrole orienté aujourd'hui à la baisse, ces deux pays restent toujours les meilleures destinations des Africains et les plus attractives pour les investisseurs dans les commerces de grande surface, dans les hôtels, les écoles privées, les cabinets des soins, et cela malgré le ralentissement de l'économie pétrolière et la situation critiques du développement sur le long terme de cette frontière. En effet, la gouvernance des communes locale, la situation des infrastructures restent encore problématique, et l'on remarque la faible ascension des nouvelles couches moyennes dans la relation Sud-Sud qui constituent néanmoins l'élite commerciale. A ces ethnies du Sud Cameroun s'ajoute une population cosmopolite venue de la sous-région Afrique Central, mais aussi de nombreux Ouest Africains. Ces migrants internationaux sont en majorité de jeunes, dont $70 \%$ sont âgés de moins de 40 ans, avec notamment une forte disparité entre les hommes et les femmes. 


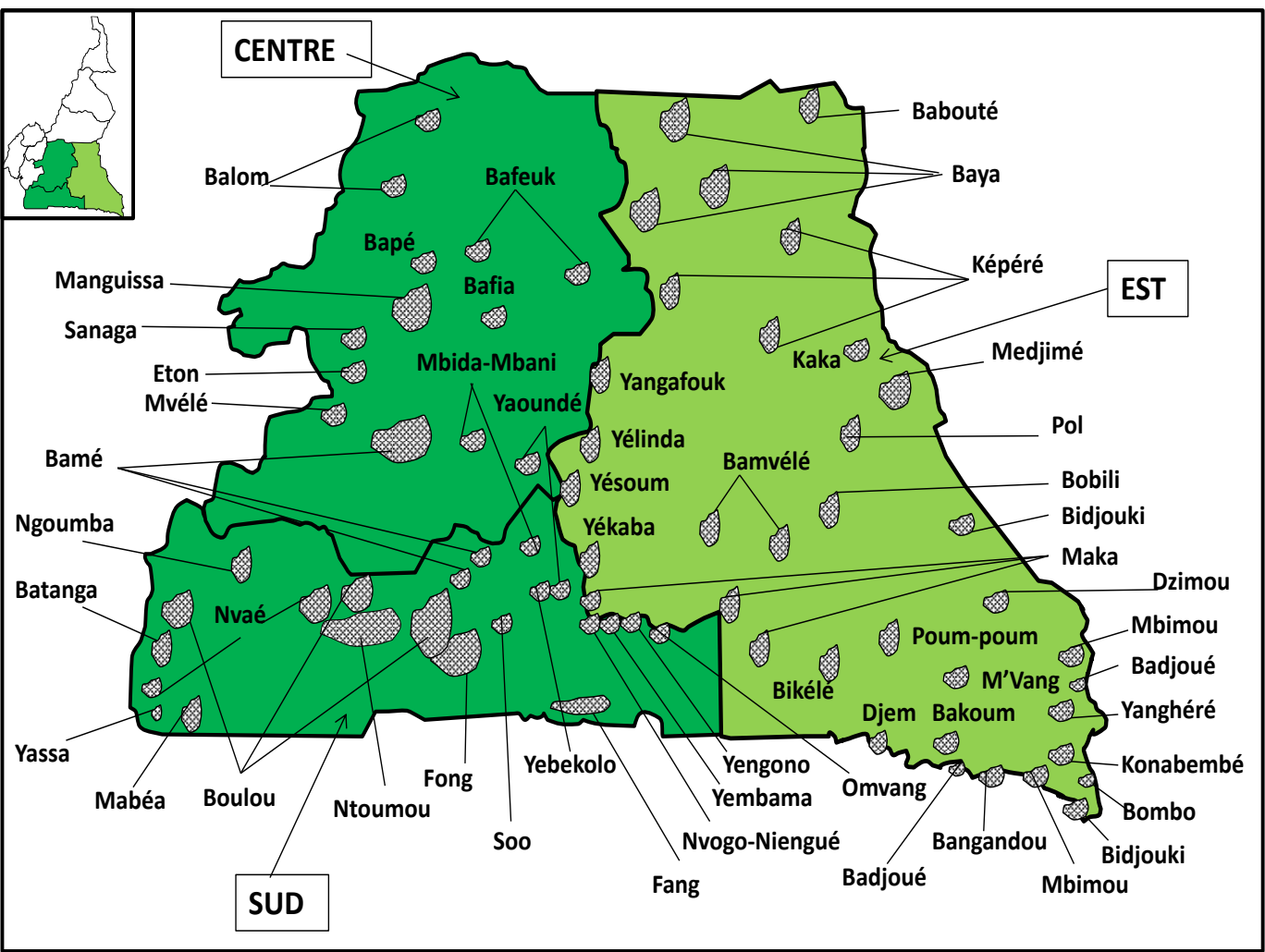

Source : Mfewou A., (2016)

Figure 1: carte des ethnies du Sud Cameroun

\section{Une dynamique transfrontalière remarquable}

A Kyé-Ossi, Abang-Minko’o et à Ebebiyin, la perméabilité des passages entre les pays est régulée à l'entrée avec un visa et vice versa, des formes de régulation sectorielles sont confié à l'autorité administrative qui est le démembrement de l'Etat, qualifiées de "gouvernance transfrontalière ». Des collaborations de travail existent entre ces communes, les chefs traditionnels, et la sous-préfecture. Il existe aussi une coopération intercommunale transfrontalière entre les communes de Kyé-Ossi, de AbangMinko'o et de Ebebiyin qui permet de discuter sur la problématique des tracasseries policières et douanières des trois côtés de la frontière, mais aussi de violences. Il faut en effet noter que les étrangers sont souvent victimes de massacres xénophobes dans l'émirat pétrolier Equato-Guinéen. L’évolution des discours actuels sur la construction de l'intégration de la sous-région évoluent et s'appuient d'abord sur l'histoire, alors que les sociétés de ces trois Etats avaient une vie commune. Les élus locaux souhaitent ouvertement les jumelages des communes, pour se situer au niveau de la coopération entre les collectivités locales et solliciter des dynamiques multilatérales par la construction d'autoroute et voire même d'un chemin de fer transnational. Par 
exemple, le Réseau des femmes actives de la Cemac (REFAC) a organisé récemment la Foire transfrontalière annuelle de la Cemac 2016 (Fotrac) à KyéOssi, sous le thème : «agir pour la paix, le développement durable, l'intégration régionale pour l'émergence de l'Afrique».

\section{Des dynamiques de développement local remarquables}

Des activités marchandes et agricoles se déroulent aux confins forestiers du Sud Cameroun. Cette frontière est ensuite l'objet de représentations diverses de la part d'une multiplicité d'acteurs. La diversité des pratiques commerciales est à l'origine de la construction d'un espace marchand aujourd'hui appelé «marché mondial». Cet espace marchand couvre la presque totalité de la ligne frontalière au Sud du Cameroun (Figure 2). Dans cette immense frontière s'est construit des marchés dits frontaliers, dont les plus importants sont Kyé-Ossi et Abang-Minko'o dans la partie camerounaise et Ebebiyin au-delà des frontières du Cameroun. La mise en relation de ces espaces marchands de part et d'autre de la frontière camerounaise est à l'origine de dynamiques spatiales (figure 3), support des mutations commerciales. Dans cette partie, le commerce reste une activité quasiment en plein temps (6h-18h30), pratiquée par plusieurs acteurs (producteurs, grossistes, semi-grossistes, intermédiaires et détaillants) qui en tirent l'essentiel de leurs revenus, soit en moyenne 20000-50000Fcfa ${ }^{29}$. Ces acteurs commerciaux ont effectué un parcours à l'école avant d'abandonner $(91,75 \%)$, et cette formation scolaire joue un rôle important dans les négociations et le savoir-faire commercial (tableau 1).

Avec une forte présence des femmes (66,88\%), l'efficacité de la commercialisation est considérée à partir de la satisfaction des consommateurs en termes de disponibilité régulière des produits agricoles et de l'abondance des produits forestiers (tableau 2). Il faut signaler que la consommation varie selon les différents facteurs socio-économiques : la taille du ménage (3$5 \mathrm{membres} / \mathrm{ménage}$ ); le revenu faiblement représenté pour la population locale camerounaise et le revenu salarial hautement élevé pour les membres des familles gabonais et équato-guinéen grâce à la manne pétrolière. Les caractéristiques socioculturelles des groupes ethniques sont également importantes, avec ceux qui consomment plus de la banane plantain ou du manioc ou des produits forestiers non ligneux qui sont des indicateurs de différenciation indispensables pour la typologie des ménages rencontré entre ces trois pays.

${ }^{29} 1 \mathrm{USD}=554,64815 \mathrm{XAF}$ 
Tableau 1 : Niveau académique des acteurs commerciaux agricole

\begin{tabular}{|c|c|c|c|}
\hline \multirow[b]{2}{*}{ Niveau Scolaire } & \multicolumn{2}{|c|}{ sexe } & \multirow[t]{2}{*}{ Total } \\
\hline & Hommes & Femmes & \\
\hline Primaire & 9,55 & 20,96 & 30,52 \\
\hline Secondaire & 18,96 & 39,34 & 58,30 \\
\hline Universitaire & 2,34 & 0,59 & 2,93 \\
\hline aucun & 2,26 & 5,98 & 8,24 \\
\hline
\end{tabular}

Source : Nombre de commerçants agricoles enquêtés pendant l'étude (2016)

Tableau 2 : Acteurs commerciaux agricole par âges et par sexe aux Confins Sud-Cameroun

\begin{tabular}{cccc}
\hline âge & \multicolumn{3}{c}{ sexe } \\
\cline { 2 - 4 } & Hommes & Femmes & Total \\
\hline$<18$ ans & 7,69 & 5,09 & 12,79 \\
$\geq 18$ ans & 25,42 & 61,78 & 87,21 \\
\hline Total & 33,12 & 66,88 & 100 \\
\hline
\end{tabular}

Source : Nombre de commerçants enquêtés pendant l'étude (2016)

Il ressort de ces tableaux (1 et 2) que les femmes sont fortement représentées dans les activités commerciales. Il faut noter que la typologie des produits agricoles commercialisés est d'avantage fonction des habitudes alimentaires de la population (tableau 3). La banane plantain, le manioc, le haricot, la tomate, le bâton de manioc, l'arachide, l'oignon, les légumes, le tubercule, et les produits forestiers non ligneux sont des produits fortement rencontrés sur ces marchés frontaliers. Toutefois, le prix par rapport au budget alimentaire de ménage est en moyenne de 10000-2000Fcfa/journalier/ménage. Aussi de la disponibilité régulière et de la qualité du produit sont ravitaillés par des grossistes venus de l'intérieur des grands bassins de productions camerounais. Les prix des produits font l'objet de très fortes variations saisonnières. Ces produits coûtent plus chers en saison sèche, par exemple le prix de la banane plantain augmente en cette période entre 5000$10000 \mathrm{Fcfa} /$ régime et de nombreux consommateurs délaissent cette denrée. Parfois les routes sont impraticables en saison pluvieuse et pénalisent l'ensemble des activités.

Tableau 3 : Principaux produits agricoles transfrontaliers du Sud-Cameroun-Gabon-Guinée Equatoriale

\begin{tabular}{cc}
\hline Marché transfrontaliers & Produits agricoles \\
\hline Kyé-Ossi & Banane plantain, manioc, haricot, tomate, bâton de \\
Abang-Minko'o & manioc, arachide, oignon, légumes, tubercule, et \\
et à Ebebiyin. & produits forestiers non ligneux \\
\hline
\end{tabular}


Tableau 4 : Typologie d'acteurs commerciaux agricoles

\begin{tabular}{cccc}
\hline Types d'acteurs & sexe & & Total \\
\cline { 2 - 4 } & Hommes & Femmes & \\
\hline Grossistes & 1,977 & 10,13 & 12,10 \\
Semi grossistes & 3,64 & 22,83 & 26,47 \\
détaillants & 25,16 & 33,45 & 58,61 \\
Intermédiaires & 2,34 & 0,476 & 2,81 \\
\hline
\end{tabular}

Source : Nombre de commerçants enquêtés pendant l'étude (2016)

Tous les produits commercialisés dans ces marchés frontaliers (KyéOssi Abang-Minko'o et à Ebebiyin) passent par un stade de vente au détail alors que le stade de vente en gros est parfois systématique à l'arrivée dans des camions très tôt le matin $(5 \mathrm{~h})$ où par les semi-grossistes et des intermédiaires qui discutent le chargement pour revendre aux détaillants $(58,61 \%)$. Par ailleurs, les intermédiaires $(2,81 \%)$ connaissent généralement les zones de production agricole et ils informent parfois les grossistes de l'emplacement du champ pour qu'ils puissent négocier rapidement l'achat pré-récolte directement avec les producteurs.

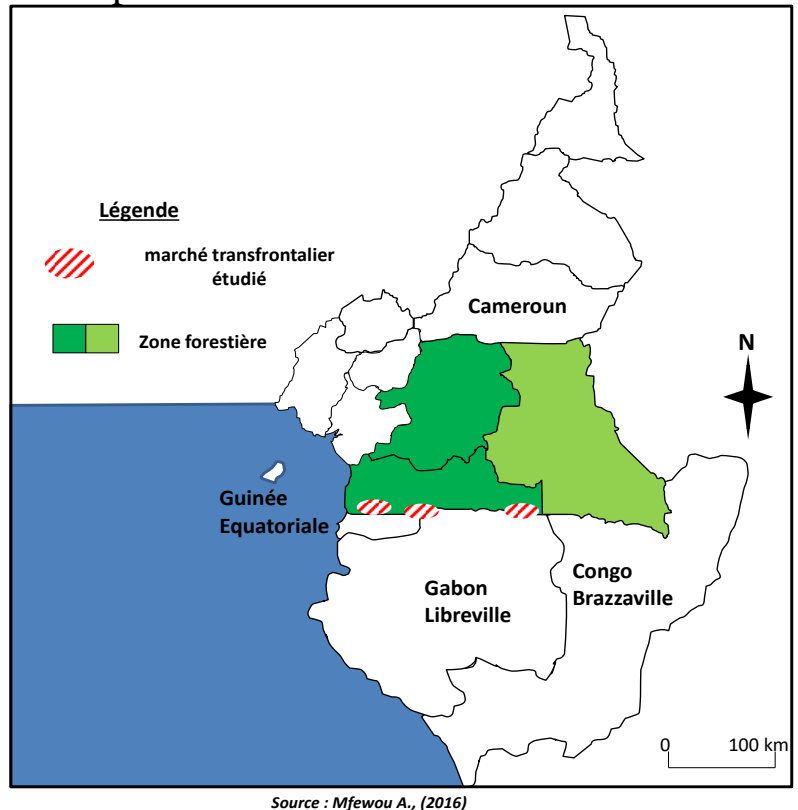

Figure 2 : Frontière commerciale entre le Cameroun et ses voisins du Sud 


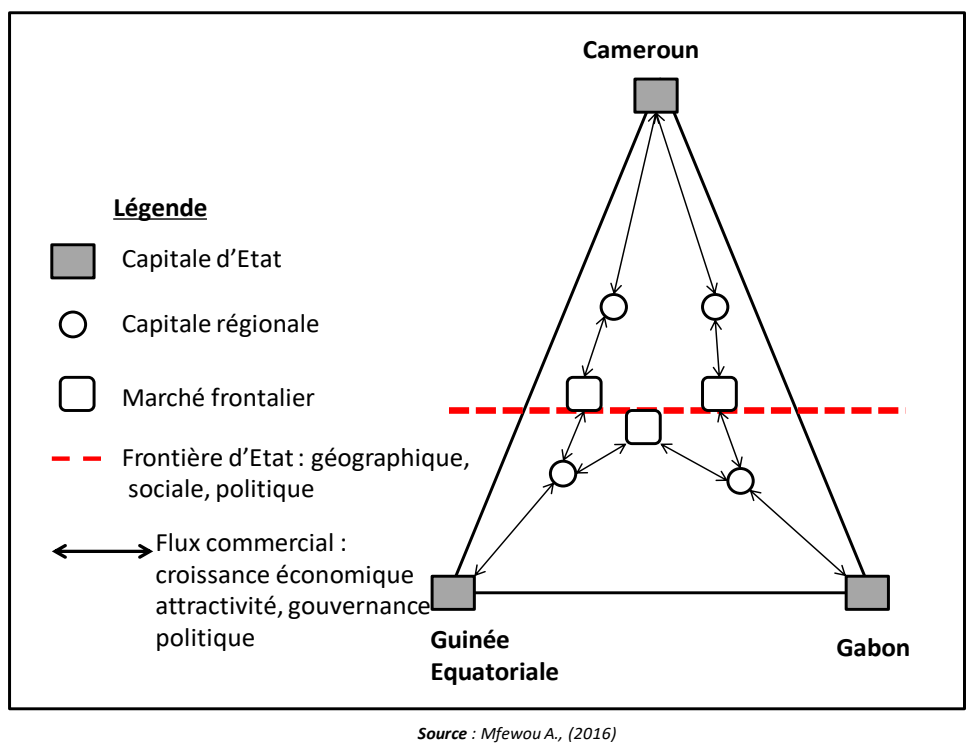

Figure 3: Structuration spatiale : dynamiques sociales et transfrontalière en zone forestière

L'amplification de ces échanges marchands conjointement à l'exploitation des différentiels frontaliers et des complémentarités agroécologiques entraine des mutations territoriales dans les espaces frontaliers du Cameroun, de la Guinée Equatoriale et du Gabon. Dans cet espace marchand créé de part et d'autre de la frontière camerounaise se construisent des jeux d'échange commerciaux avec du côté camerounais, la mise à disposition des acheteurs de produits agricoles et non agricoles venus principalement de l'hinterland du Cameroun. A cela s'ajoute la commercialisation de certains produits importés via le port de Douala comme le riz, la farine et autres produits manufacturés, mais aussi des produits forestiers l'Okok, le gibier, pour ne citer que ces exemples.

Par ailleurs, le Gabon et la Guinée Equatoriale importent le bétail camerounais dans le cadre d'une réglementation de la CEMAC qui régit sa circulation à l'intérieur de la sous-région (Mfewou et al., 2016). Dans cette activité économique liée au bétail (Bennafla 2002; Corniaux et al. 2012), la commercialisation des animaux connait un léger développement à Kyé-Ossi, Abang-Minko'o et Ebebiyin au cours de ces dix dernières années. Mais sa commercialisation se heurte encore à la précarité à des conditions de transport (problèmes de camions et d'infrastructures routières) et aux barrages routiers qui imposent des taxes dont on cerne aussi difficilement l'origine. 


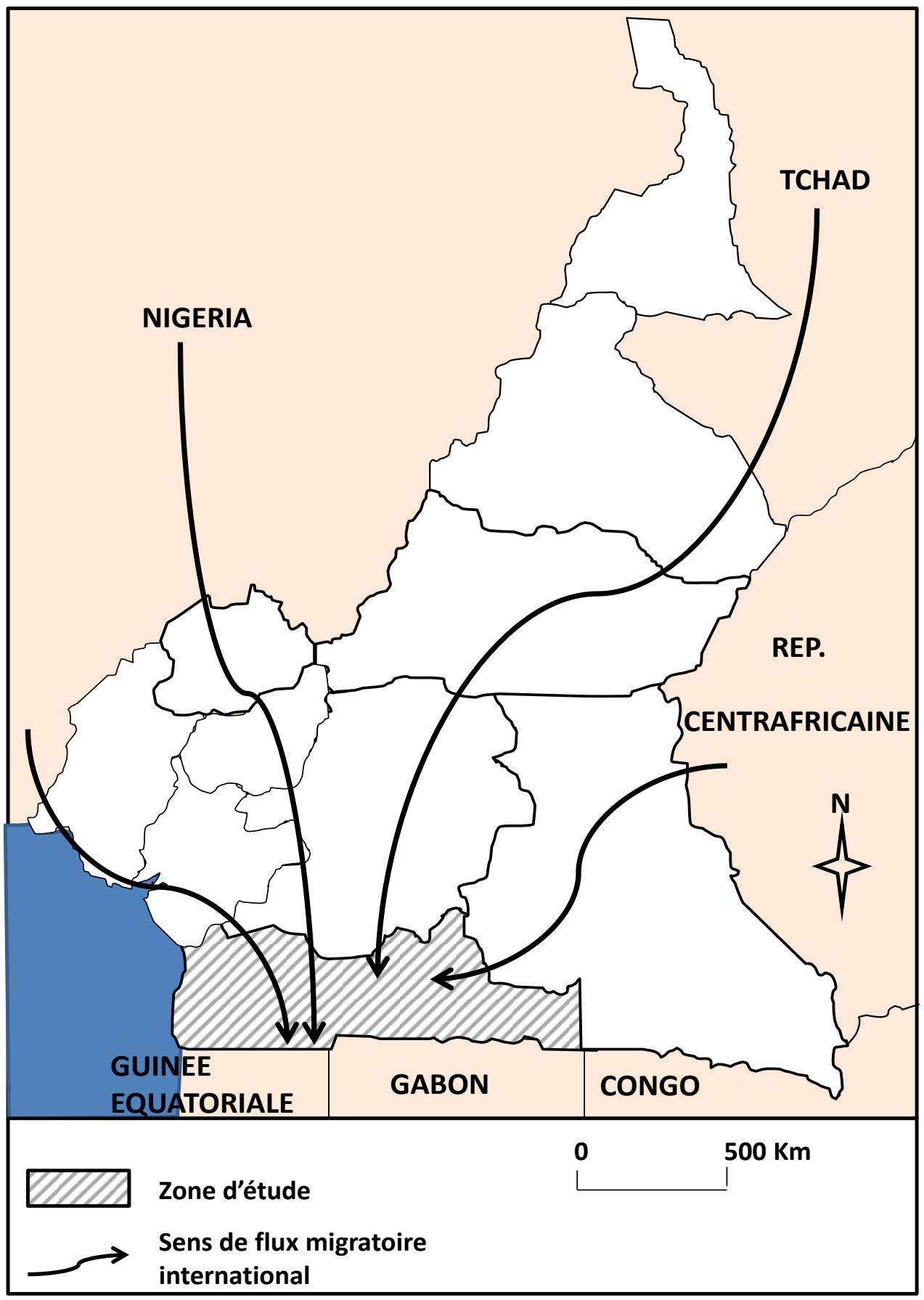

Source : Mfewou A., (2016)

Figure 4 : Sens de flux migratoire international à la frontière Sud-Cameroun

Il se met donc en place une multitude de marchés quotidiens des centres petits-urbains frontaliers plus fréquenté surtout en matinée. C'est ainsi 
que Kyé-Ossi, Abang-Minko'o et Ebebiyin se sont affirmés comme des plaques tournantes commerciales fondamentale entre le Cameroun, la Guinée Equatoriale et le Gabon. Ces marchés sont ainsi devenus les principaux pôles de ravitaillement des villes capitales que sont Libreville et Malabo. C'est ainsi que ces marchés font l'objet des attractivités au-delà de la sous-région, avec l'arrivé quasi des migrants-commerçants venus de l'Afrique de l'Ouest (nigérian, malien, sénégalais,...), qui au-delà des activités commerciales classiques s'investissent pour certains dans les activités de débrouillardises pour d'autres comme la réparation des chausseurs, les tâches ménagères ou conduite moto taxi... On assiste donc à un important brassage de populations autochtones et étrangères.

Les mutations du secteur commercial, avec surtout la nécessité d'associer l'hinterland du Cameroun (zone productrice des denrées agricoles), mais aussi les dynamiques démographies et le brassage des cultures ont eu un impact significatif sur le secteur agricole local. L'intérêt pour une agriculture de proximité s'est en effet accru dans la région, surtout dans la localité de KyéOssi au cours de ces dix dernières années bien que le ravitaillement continue à se faire à partir de l'intérieur du Cameroun. Cette agriculture péri-frontalière est pratiquée surtout par les agriculteurs-maraichers venus de la région de l'Ouest-Cameroun installé à Kyé-Ossi et qui ont développé ce système d'économie pour réduire le coût de transports d'habitude distant des grandes zones de production de l'Ouest-Cameroun.

Deux phénomènes expliquent en partie cet emplacement de proximité direct avec les consommateurs : la rapidité de la croissance d'attractivité frontalière, d'une part, la gouvernance locale des politiques foncières dont les paysans sont parfois confrontée à une insécurité foncière agricole aiguë qui est à la fois le reflet d'une complexité de l'histoire locale et de conflits de droits d'usage foncier ou du droits de propriété dans un contexte d'intense pression foncière. Il faut aussi noter le phénomène de l'hospitalité de la population locale. Les trois pays interagissent entre eux. Ainsi, les villes capitales du Gabon (Libreville) et Guinée Equatoriale (Malabo) influence sur les zones des productions de Kyé-Ossi en favorisant l'émergence de secteurs maraichers (tomate, légumes, condiments...) marchands à la frontière à produire plus.

Avec les connexions spatiales mise en place autour des marchés (figure 2), la perméabilité des passages est régulée à l'entrée avec un visa, dans les deux sens, des formes de régulation sectorielles sont confié à l'autorité administrative qui est le démembrement de l'Etat, qualifiées de « gouvernance transfrontalière ». La collaboration de travail existe entre ces communes, les chefs traditionnels, et la sous-préfecture. Il existe aussi une coopération intercommunale transfrontalière entre les communes de Kyé-Ossi, de AbangMinko'o et de Ebebiyin, c'est aussi revenir sur la problématique des 
tracasseries policière, douanière des trois côtés «Les étrangers sont souvent victimes de menace des étrangers dans l'émirat pétrolier Equato-Guinéen ».

L'évolution des discours actuels sur la construction de l'intégration de la sous-région évoluent et s'appuient d'abord sur le passé historique de vie commune des trois Etats. Les élus locaux qui souhaitent ouvertement les jumelages des communes, pour se situer au niveau de la coopération entre les collectivités locales en sollicitant les dynamiques multilatérales par la construction d'autoroute et voir même de chemin de fer transnational. Par exemple, récemment le Réseau des femmes actives de la Cemac (REFAC) a organisé la Foire transfrontalière annuelle de la Cemac 2016 (Fotrac) à KyéOssi, sous le thème : «agir pour la paix, le développement durable, l'intégration régionale pour l'émergence de l'Afrique».

\section{Dynamique sociale et Sécuritaire à la frontalière}

Dans une évolution sociale, à travers le vivre ensemble de plusieurs acteurs d'origines différents et surtout la mise en place de connexion de réseaux de communication (route, téléphonie...) parfois problématique, l'on observe une intégration académique avec la création multiple écoles et lycées à Kyé-Ossi, à Abang-Minko'o et à Ebebiyin. Ces biens sociaux tissent les liens de solidarités quotidiennes entre les familles de la région à travers les associations des parents d'élèves, ce lien constitue l'un des facteurs d'intégration permettant de renforcer la cohésion sociale (Ngodi, E., 2005; Bouquet C., 2003 et Bach D. C., 1998).

Mais, la libre circulation des personnes et des biens est loin d'être réglée entre ces États, lié aux difficultés de la mise en pratique des traités d'ouverture des frontières signés entre les États depuis le 16 mars 1994 qui institue la Communauté économique et monétaire de l'Afrique centrale (CEMAC), la convention du 5 juillet 1996 affirme que « l'union constitue un seul territoire douanier à l'intérieur duquel la circulation des personnes, marchandises, biens, services, et capitaux est libre» (Messe Mbega, C. Y., 2015; Magrin, G., 2003; Mfege, A. H. O., 2005).

La sécurité et la défense restent aujourd'hui les grands défis actuels avec cette population composite et évolutive, le renforcement des pratiques de la sécurité nationale au-delà des frontières nationales avec le contrôle mixte, les surveillances et d'identification à l'entrée et à la sortie des trois Etats sont permanents.

\section{Conclusion}

Nos travaux illustrent bien la conjugaison nécessaire d'un certain nombre de facteurs pour qu'émerge un espace de croissance périphérique, structuré par un ou plusieurs marchés frontaliers d'envergure "marché mondial ». Le pétrole dans cette partie de l'Afrique est le grand initiateur de 
cette mutation transfrontalière caractérisée par une dynamique sociale et spatiale dans la zone forestière du Cameroun. Notre analyse a permis de mieux appréhender la diversité des potentialités en termes d'intégration des économies, des territoires et des sociétés venues de toute l'Afrique. Elle a aussi mis l'accent sur l'émergence de priorités comme la construction des grands réseaux routiers, la construction de l'énergie électrique, la régulation du marché. Celles-ci sollicitent le rôle du politique et sont indissociables du dialogue intercommunal dans une décentralisation effective sollicitée par les acteurs. Il faut bien sûr noter que la mise en place d'une gouvernance à l'occasion de la décentralisation des pouvoirs de l'Etat reste un défi et est encore une question d'actualité dans cette partie de l'Afrique. Ces réseaux viendront renforcer la dynamique sociale et économique.

Bien que riche du pétrole, la Guinée et le Gabon restent dépendants des produits agricoles de l'extérieur pour leur population. Ainsi, la périphérie camerounaise connaît des logiques de dépendance vis- à-vis des espaces voisins, en termes d'attractivité des pôles étrangers pour le développement de segments économiques, parfois compétitifs entre acteurs.

Le développement du commerce transfrontalier a également «dynamisé les activités maraîchères de proximité dans les bas fond du Sud Cameroun en leur offrant un emploi sûr pour les paysans de cultiver et écouler rapidement ces produits aux consommateurs locaux et étrangers.

\section{References:}

1. Bach, D. C. (1998). Les dynamiques paradoxales de l'intégration en Afrique subsaharienne: le mythe du hors-jeu (Vol. 3). Centro de Estudos Internacionais do Instituto Universitário de Lisboa (ISCTEIUL).

2. Bennafla, K. (2002). Le commerce frontalier en Afrique centrale: acteurs, espaces, pratiques. KARTHALA Editions.

3. Bouquet, C. (2003). L'artificialité des frontières en Afrique subsaharienne. Turbulences et fermentation sur les marges. Les Cahiers d'Outre-Mer. Revue de géographie de Bordeaux, 56(222), 181-198.

4. BUCREP, (2010). Troisième recensement général de la population et de l'habitat ( $\left.3^{\mathrm{e}} \mathrm{RGPH}, 2005\right)$, Bureau central des recensements et des études de population du Cameroun.

5. Corniaux, C., Thébaud, B., \& Gautier, D. (2012). La mobilité commerciale du bétail entre le Sahel et les pays côtiers: l'avenir du convoyage à pied. Nomadic Peoples, 6-25.

6. Diallo, M. M. (2014). Dynamiques transfrontalières et stratégies d'aménagment du territoire en Afrique de l'Ouest: analyse à partir des 
frontières Sénégal-Gambie et Sénégal-Guinée Conakry. Territoires d'Afrique, (6), 33-43.

7. Hugon, P. (1989). Les stratégies comparées des pays africains du golfe de Guinée à l'épreuve du contre-choc pétrolier. Revue Tiers Monde, 755-778.

8. Chauveau, J. P., Jacob, J. P., \& Le Meur, P. Y. (2004). Gouverner les hommes et les ressources: dynamiques de la frontière interne. A. Colin; IRD.

9. Christian Bouquet et Vélasco-Graciet (2007). Regards géopolitiques sur les frontières, Paris, L'Harmattan.

10. Igué, J. O. (2010). La problématique frontalière. Frontières, espaces de développement partagé, Paris, Karthala, 21-50.

11. IMF / GABON (2016): incidence économique de la chute des prix du pétrole. Country Report No. 16/87. March 2016.

12. Lévy, J., \& Lussault, M. (2003). Dictionnaire de la géographie [et de l'espace des sociétés] (Vol. 1034). Paris: Belin.

13. Magrin, G., Jamin, J. Y., Faure, G., \& Duteurtre, G. (2003, September). Les savanes d'Afrique centrale entre enclavement et intégration aux marchés/ In Annales de géographie (pp. 471-494). Armand Colin.

14. Messe Mbega, C. Y. (2015). Les régions transfrontalières: un exemple d'intégration sociospatiale de la population en Afrique centrale? Éthique publique. Revue internationale d'éthique sociétale et gouvernementale, 17(1).

15. Mfege, A. H. O. (2005). Le Cameroun et ses frontières: une dynamique géopolitique complexe. Editions L'Harmattan.

16. Mfewou Abdoulay, Boutrais Jean et Poutougnigni Youchahou (2016). «Dynamiques et entraves au développement du marché de bétail Ticket-Foumban dans l'Ouest du Cameroun». Revue canadienne de géographie tropicale/Canadian journal of tropical geography 25 décembre 2016, pp. 43-25.

17. Mfewou, A., Njoya A., et Oyep E., J., (2016) : Mondialisation, variabilité du commerce de bétail et de la viande bovine en Afrique Centrale. editions-ue.com

18. Ngodi, E., (2005). Gestion des ressources pétrolières et développement en Afrique. Présentation à la 11 ème Assemblée générale du CODESRIA, 6-10.

19. Nkendah, R., Ako, E., Tamokwe, B., Nzouessin, C., Njoupouognigni, M., Melingui, E., \& Azeufouet, A. (2011). Cameroun. Le commerce transfrontalier informel des produits agricoles et horticoles. Économie rurale, (4), 34-49. 